\title{
Data Analysis Method for Wind Turbine Dynamic Response Testing
}

\author{
T. L. OIsen \\ S. M. Hock
}

June 1989

Prepared for the 24th

Intersociety Energy Conversion

Engineering Conference

Washington, D.C.

6-11 August 1989

Prepared under Task No. WE911202

Solar Energy Research Institute

A Division of Midwest Research Institute

1617 Cole Boulevard

Golden, Colorado 80401-3393

Prepared for the

U.S. Department of Energy

Contract No. DE-AC02-83CH10093 


\title{
NOTICE .
}

This report was prepared as an account of work sponsored by an agency of the United States government. Neither the United States government nor any agency thereof, nor any of their employees, makes any warranty, express or implied, or assumes any legal liability or responsibility for the accuracy, completeness, or usefulness of any information, apparatus, product, or process disclosed, or represents that its use would not infringe privately owned rights. Reference herein to any specific commercial product, process, or service by trade name, trademark, manufacturer, or otherwise does not necessarily constitute or imply its endorsement, recommendation, or favoring by the United States government or any agency thereof. The views and opinions of authors expressed herein do not necessarily state or reflect those of the United States government or any agency thereof.

\author{
Printed in the United States of America \\ Available from: \\ National Technical Information Service \\ U.S. Department of Commerce \\ 5285 Port Royal Road \\ Springfield, VA 22161
}

Price: Microfiche A01

Printed Copy A02

Codes are used for pricing all publications. The code is determined by the number of pages in the publication. Information pertaining to the pricing codes can be found in the current issue of the following publications which are generally available in most libraries: Energy Research Abstracts (ERA); Government Reports Announcements and Index (GRA and I); Scientific and Technical Abstract Reports (STAR); and publication NTIS-PR-360 available from NTIS at the above address. 


\section{DATA AMALYSIS METHOD FOR GIYD TURBINE DMAMIC RESPONSE TESTING}

T. L. Olsen and S. M. Hock

Solar Energy Research Institute

Golden, CO

\section{ABSTRACT}

The Wind Research Branch at the Solar Energy Research Institute (SERI) has developed an efficient data analysis package for personal computer use in response to growing needs of the wind turbine industry and SERI'S Cooperative Field Test Program. This new software is used by field test engineers to examine wind turbine performance and loads during testing, as well as by data analysts for detailed post-processing.

The Wind Data Analysis Tool Set, WINDATS, has been written as a collection of tools that fall into two general groups. First, the preparatory tools perform subsection, filtering, decimation, preaveraging, scaling, and derivation of new channels. Second, analysis tools are used for mean removal, linear detrending, azimuth averaging and removal, per-rev averaging, binning, and spectral analysis. The input data file can be a standard ASCII file as is generated by most data acquisition software.

\section{IMTRODUCTION}

SERI's cooperative field test activities have resulted in large quantities of data, and we have found that the analysis of this data base is very time consuming. An assortment of data processing tools is available, including commercial software, but the time and effort required to apply them to large quantities of data are often restrictive. We have determined that our data analysis requirements would be better met by a software tool tailored specifically to our needs. The goal of this work, therefore, is to streamline and simplify our data processing capability with emphases on consolidation, speed, efficiency, accuracy, and flexibility. This paper begins with a brief review of the U.S. Department of Energy's (DOE) Cooperative Field Test Program and then presents the resulting data analysis method.

\section{COOPERATIVE FIELD TEST PROGRAM}

Over the past several years, DOE has sponsored a series of cooperative tests with the wind energy industry to provide a better understanding of both the operating environment and operating characteristics of wind energy conversion systems. SERI has been intimately involved with most of these tests by coordinating test planning, providing test equipment, and providing test engineering support to help run the field tests. Typically, the wind turbines are heavily instrumented with sensors to measure the dynamic responses of the machines to wind inflow. The wind itself is also measured in detail, often with a vertical plane array of anemometers located upwind of the turbine to provide wind data over the plane of rotation.

Figure 1 shows one test turbine, the Northern Power Systems (NPS) 100-kW wind turbine, on which field tests were performed during the summers of 1987 and 1988. The NPS 100-kW "North Wind 100" wind turbine has a two-bladed, upwind, teetered rotor employing a passive pitch rotor control system. The 17.8-m diameter rotor has two fiberglass blades and is located at a hub height of $25.6 \mathrm{~m}$, atop a tapered steel tubular tower.

The machine was located in Altamont Pass, Calif. The test program objectives were to acquire and document the operating loads and performance parameters of the turbine, and provide a detailed view of the wind characteristics for correlation with

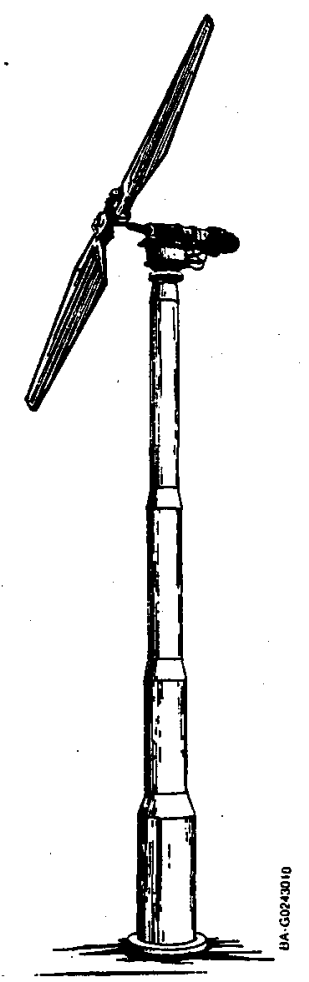

FIGURE 1 
the loads and performance data. Eighty-two channels of data were recorded in multiplexed form on a 16-track magnetic tape for analysis. The following discussion uses a small portion of these data to demonstrate the capabilities of the data analysis method.

\section{COMPUTATIONAL REEDS}

Most of the tools mentioned earlier are used at SERI. However, they are scattered throughout separate programs that are quite useful by themselves but are not designed with a standardized beginningto-end data analysis path in mind. Thus, it is awkward and time-consuming to analyze the large amounts of wind turbine field test data and present them in a meaningful form to other researchers. A typical 10 -min data set would take perhaps $6 \mathrm{hr}$ to process. In addition, the tools are not readily available for use at the field test sites to produce timely feedback, which is necessary to ensure quality data and successful testing. Therefore, computational requirements include flexibility, transportability, processing time reduction (less than 1 hr total for a 10-min data set), batch processing (a single program in which all operations can be run without intermittent human attention), and the use of a commonly available computing environment (such as a personal computer).

Speed, batch processing, and flexibility are achieved by using an interactive front-end processor to set up a control file for the data reduction. Transportability is limited only by the minimum computer requirements of $640 \mathrm{~K}$ RAM with a math coprocessor, a PC-DOS version 2.0 or higher, and a FORTRAN 77 compiler if the source code requires modification. The analysis package accepts data files of any length, ASCII or binary, volts (counts), or engineering units, and a maximum of 90 channels in any order. Intermediate files are saved for future access. Two program units are used. The spectral processor (WINSPEC) is separate from the time-series analysis package (WINDATS). This separation alleviates program memory size problems and allows for planned expansion of the spectral analysis module. Additional flexibility is achieved, and user selectivity is possible at a critical point in the analysis. Basic flow charts for the two programs are shown in Figures $2 a$ and $2 b$, and a sample process control file is presented in Figure 3.

\section{AMALYSIS TOOLS}

The following discussion includes sample graphs showing representative results for a segment of time-series data from the recent field tests of the NPS 100-kW wind turbine previously described. The original data set contains 20 channels of data, each sampled at 25 samples per second. An example of one channel of the original time series data sampled at $25 \mathrm{~Hz}$ for the entire $11 \mathrm{~min}$ is shown in Figure 4. This plot represents the blade root flapwise bending moment. It is apparent from Figure 4 that the signal contains information over a wide range of frequencies, but it is difficult to quantify characteristics such as long-term trends and

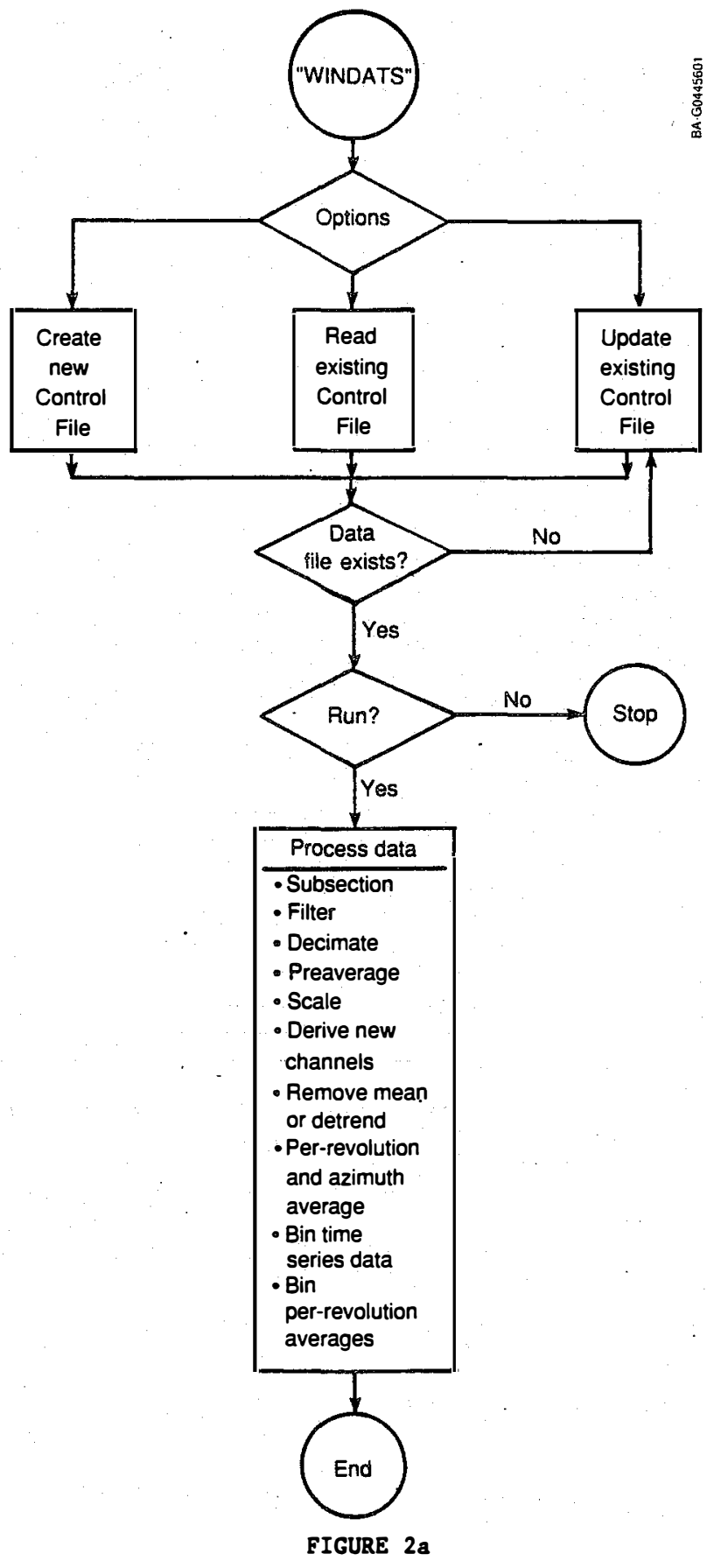

periodicity of the signal. Figure 5 shows the same signal over a much shorter time interval $(10 \mathrm{sec})$. Here the periodicity of the signal can be seen with much more detail. The data analysis program will help correlate the behavior of this bending moment with other measurements such as the wind inflow and the blade position by applying the data reduction and analysis techniques described below. 


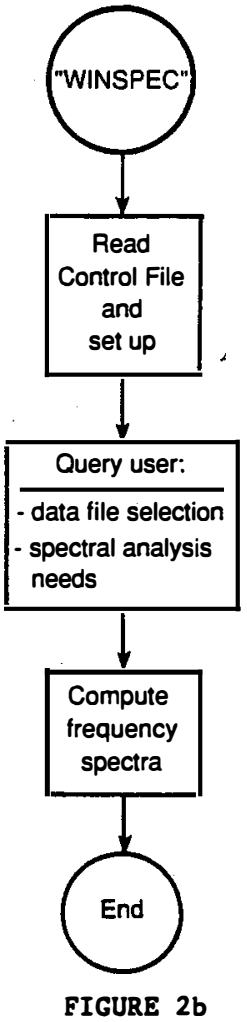

Data Preparation

Analog data collected in the field are passed through analog filters before being sampled at a rate consistent with the bandwidth of interest. An input data file often contains channels that have been filtered at different cut-off frequencies, depending on analysis requirements and sensor limitations. Sometimes, the recorded sample rate is several times higher than that needed for initial data analysis. In order to reduce these large volumes of data to a more manageable size without introducing aliasing from lower sample rates, the program includes modules to filter raw data and reduce bandwidth requirements, and then to decimate the data at user-specified intervals. A low-pass Butterworth filter (recursive - Infinite Impulse Response, single-zero, multipole, cascaded) is used in WINDATS, with the convention

$$
y(i)=B 0 \star_{x}(i)-A 1 \star_{y}(i-1)-A 2 \star_{y}(i-2) .
$$

It is faster than a comparable nonrecursive (Finite Impulse Response) filter in most cases, although it is sometimes unstable with more than two poles. Two poles are usually sufficient, but six are often preferred for a very sharp rolloff. (This is a user option.) The discontinuous blade azimuth (sawtooth) signal is converted to a half-angle cosine function for full $360^{\circ}$ coverage when filtering, so that it receives the same phase shift as the rest of the data. In decimation, every nth scan is retained, and the rest are discarded. Figure 6 shows the original time-series data shown in Figure 4 after being filtered at $0.5 \mathrm{~Hz}$ and
DATA DESCRIPIION:

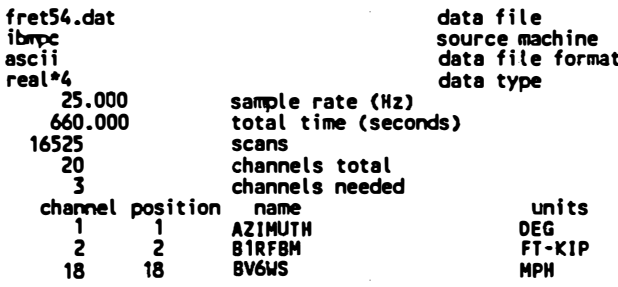

oAta preparation neEos:

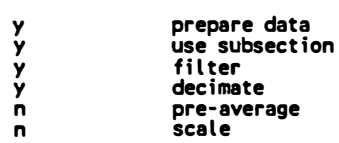

DATA PREPARATION SPECIFICATIONS:

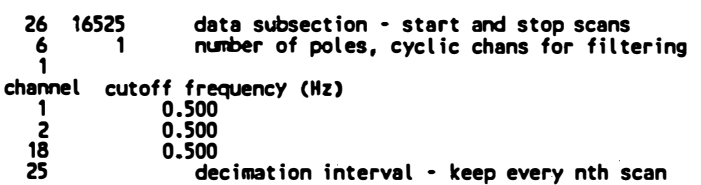

DeRIVED CHANNEL MEEDS:

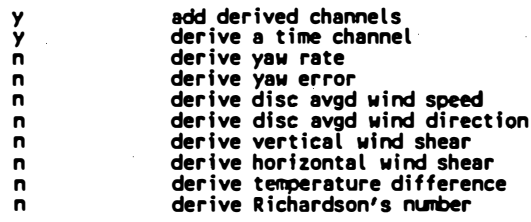

DATA ANALYSIS MEEOS:

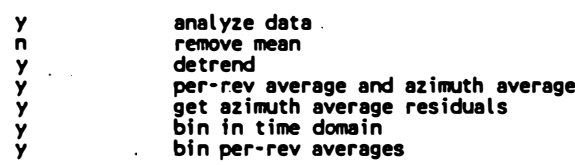

DATA ANALYSIS SPECIFICATIONS:

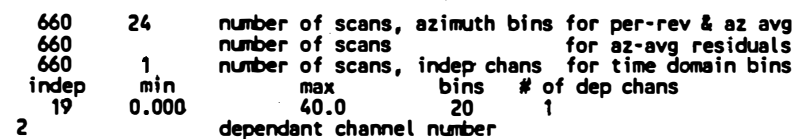

FIGURE 3

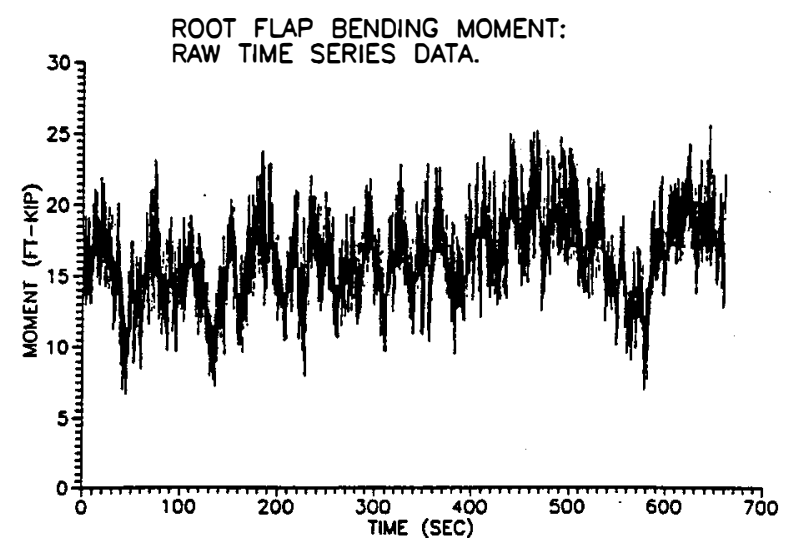

FIGURE 4 


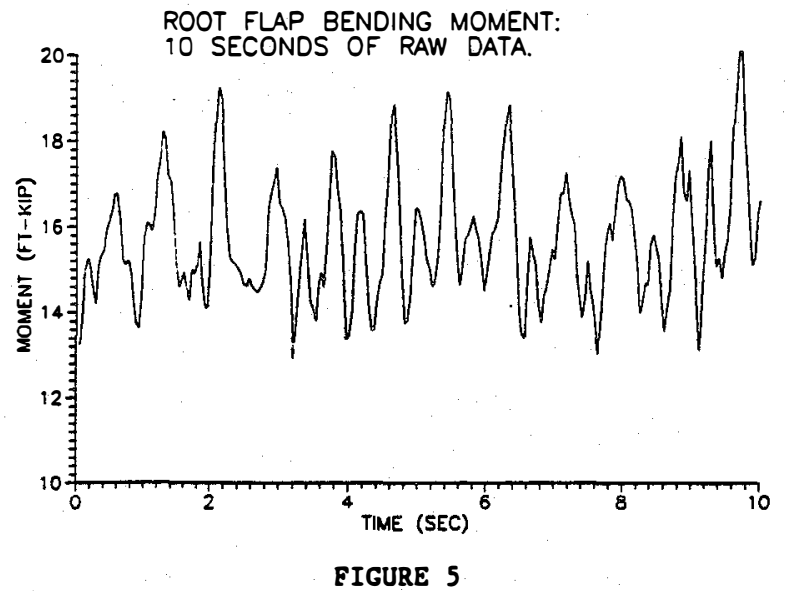

decimated by a factor of 25 to a sample rate of $1 \mathrm{~Hz}$. Visual comparison of the two plots clearly shows the reduction of high-frequency information.

Preaveraging is a crude form of filtering and decimation: Every n consecutive scans are replaced by one scan of averages for the group. It is not recommended because it may introduce some errors through aliasing, yet it is preferred occasionally. Consequently, it is an option in WINDATS as well.

The scaling operation converts measurement units (counts or volts) to engineering units. Slopes and intercepts must be specified in the control file for each channel in order to be applied to the original time-series data. A maximum value can be specified for cyclic data (such as blade azimuth or yaw angle) to cause a modulus reduction to the first cycle range $\left(0^{\circ}\right.$ to $\left.360^{\circ}\right)$. This is necessary for angular position sensors with redundant rariges such as $360^{\circ}$ to $720^{\circ}$ and $0^{\circ}$ to $-360^{\circ}$.

Once this preprocessing is complete, the derivedchannels option can create new channels that are functions of the original channels. The derived channels currently available are:

- time (scan \$/sample rate),

- yaw rate (delta yaw angle/delta time),

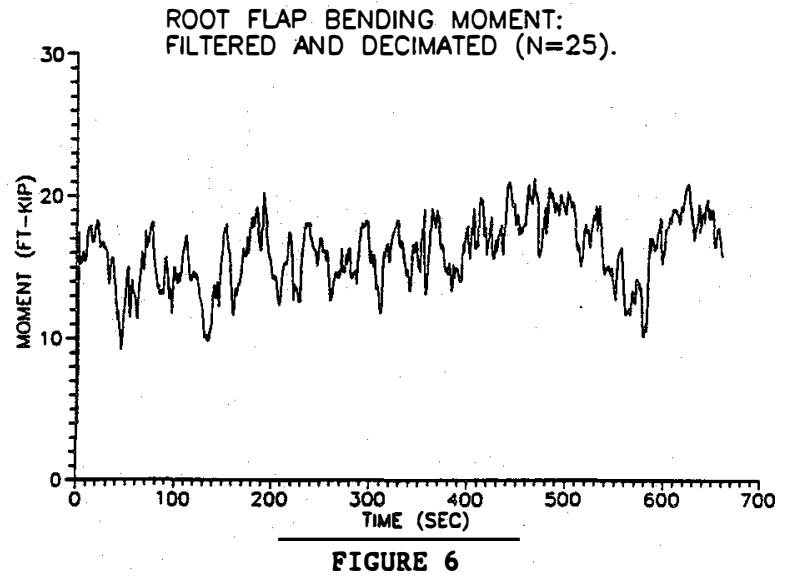

\author{
- yaw error (yaw angle - wind direction), \\ - disc-averaged wind speed and direction, \\ - vertical and horizontal wind shears, \\ - vertical temperature difference, and \\ - Richardson's number.
}

New derived channels can be added to this option as the need arises.

After each full pass through the data, statistics are computed for each channel in the resulting data file. These statistics include mean, standard deviation, and minimum and maximum values over the time segment.

\section{Data Analysis}

The analyst is often interested in examining only the harmonic content of a signal, so WINDATS allows the user two basic methods for removing the DC and very low frequency signal components. The first method, mean removal, is accomplished by subtracting a channel's mean over the total time segment from each of its data points:

$$
\begin{aligned}
x^{\prime}(i) & =x(i)-\overline{\bar{x}}, \\
\text { where } \bar{x} & =\sum_{i=1}^{N} x(i) / N,
\end{aligned}
$$

where $x^{\prime}(i)$ are new data points, $x(i)$ are individual data points, $\bar{x}$ is the mean, $N$ is the number of data points, and $i$ is the scan number. The second method, detrending, involves fitting a linear trend line to the data using a least squares fit, and then subtracting the line from the data:

$$
\begin{gathered}
x^{\prime}(i)=x(i)-y(i), \\
\text { where: } y(i)=A x(i)+B,
\end{gathered}
$$

where $A$ and $B$ are the trend coefficients, and $y$ represents the linear fit to the data.

It is assumed that for stationary operating conditions of the test turbine, deteministic and stochastic portions of the signals can be separated by the process of azimuth averaging. Azimuth averaging is accomplished by binning: dividing the rotor azimuth range into several equal groups and assigning data points from numerous rotor cycles to their respective groups. WINDATS accepts a userspecified number of groups (bins). Mean values and standard deviations for each channel are determined for corresponding data sets that fall in each bin:

$$
\begin{gathered}
\bar{y}(k)=\sum_{i=1}^{N} y[x(i)] / N(k) \\
\text { for } x \min (k) \leq x(i)<\operatorname{xmax}(k),
\end{gathered}
$$

where $i$ is the scan number, $k$ is the bin number, $x(i)$ are azimuth points, xmin and xmax define the range of bin $k, N$ is the number of data points in that bin, $y[x(i)]$ are data points corresponding to 
azimuth $x(i)$, and $y$ is the mean for bin $k$. The azimuth averaged root flap bending moment is shown in Figure 7. Notice the characteristic smooth signal shape. This signal represents the deterministic bending moment that occurs with every rotor revolution and is independent of wind turbulence.

The random loads, which are superimposed on this deterministic part of the time-series data, also contain useful information for examining the rotor loads and their contribution to fatigue damage. Residuals, which represent this stochastic portion of the signal, are produced when the azimuth average is subtracted from the original data, cycle by cycle:

$$
y^{\prime}(i)=y[x(i)] \bar{y}(k)
$$

for $x(i)$ in bin $k$, where $y^{\prime}(i)$ are the residual data points. The random nature of these residuals is apparent in Figure 8.

Per-rev averaging, which is defined as the sum of all samples for a signal over one blade revolution divided by the number of samples contained in that revolution, reduces a column of data so that a single average represents each rotor revolution. This method is useful as another way to remove higher frequency variations in a signal that occur over each cycle, to show long-term trends in the

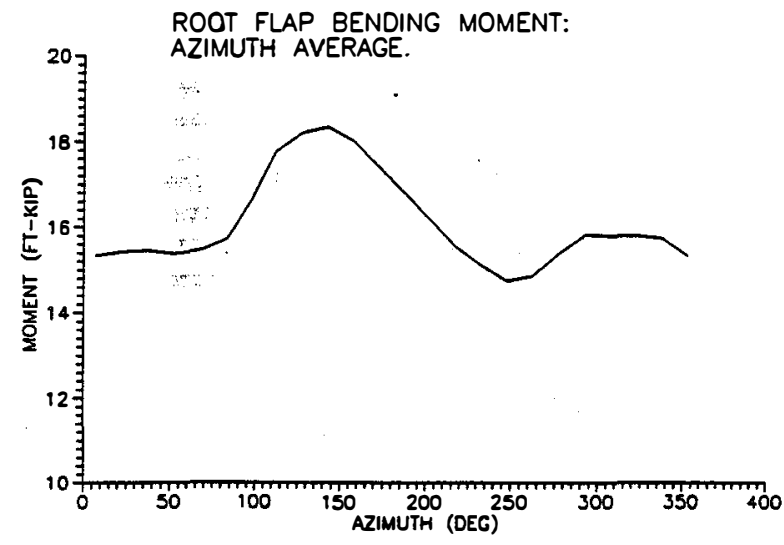

FIGURE 7

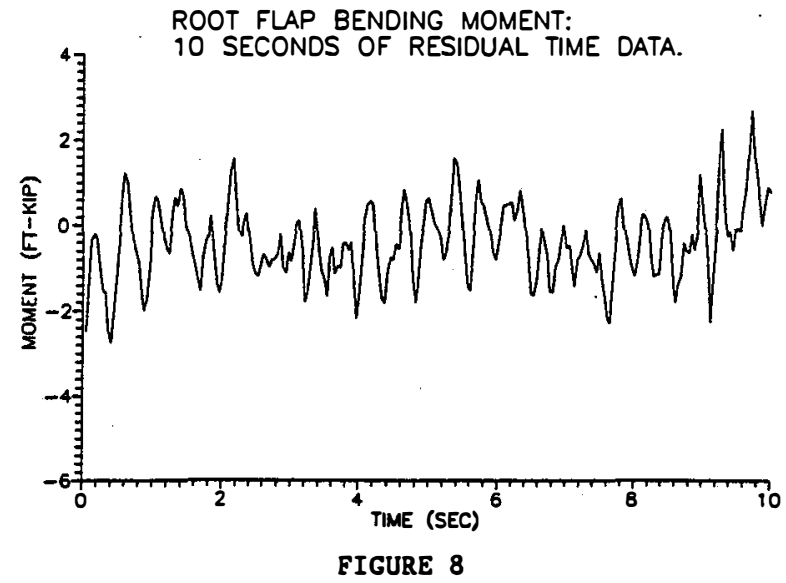

data, and sometimes to facilitate binning. It is essentially the same as preaveraging with a onerevolution block size triggered by the rotor azimuth signal.

Binning is a simple technique used in place of regression analysis to characterize the interdependency between any two channels. The data models needed for regression are often discontinuous or too complex for adequate representation of wind turbine data. In binning, the independent channel is divided into a user-specified number of equal segments, or bins. Each dependent value is placed in its respective bin, then means are calculated for each bin. The formulas are the same as for azimuth averaging, but now $x(i)$ and $y[x(i)]$ are corresponding independent and dependent data points. Figure 9 shows the characteristic relationship between bending moment and wind speed that results from the method-of-bins.

Spectral analysis is useful for looking at the frequency content of time-series data. The power spectral density routine applies a square or cosine-tapered data window to segments of the timeseries data and then performs a discrete Fourier transform to multiple data segments:

$$
x(k)=(1 / N) \sum_{r=0}^{N-1} x(r) \exp (-2 \pi i k r / N)
$$

for $k=0,1,2, \ldots,(N-1)$, where $N$ is the number of values in the segment, $i$ is $\sqrt{-1}, r$ is the scan number, $x(r)$ are time-series data points, $k$ is the frequency index [freq $(k)=$ ( $k^{*}$ sample rate)/N], and $X(k)$ are Fourier coefficients (see $[1,2]$ ). The program then converts the Fourier coefficients to spectral estimates $\{X(k) * \operatorname{con} j[X(k)]\}$ and averages the resulting frequency-transformed segments together. If desired, linear or logarithmic smoothing can be applied to the final frequency spectrum. Frequency units can be specified as hertz or radians per second. The first point (at zero frequency), which represents the steady component, or the mean, normally should be dropped before plotting on a logarithmic scale since the 108 of zero does not exist. The power spectral density plot of the root flap bending moment is

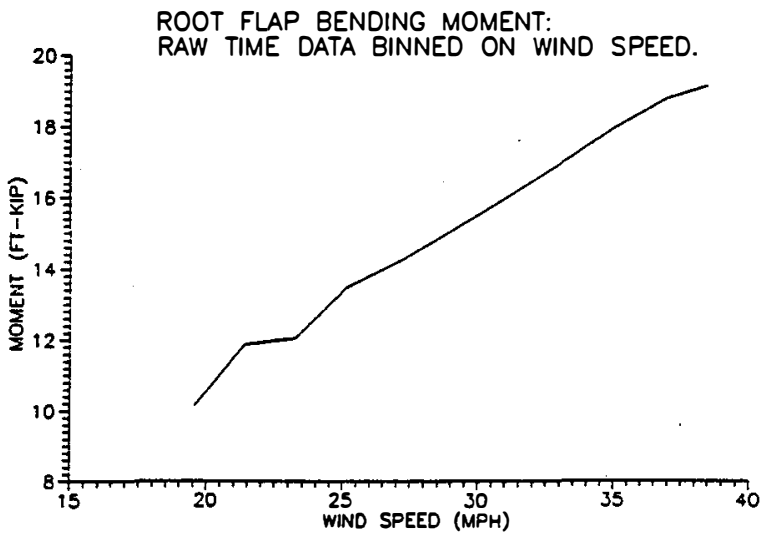

FIGURE 9 
shown in Figure 10. The large peaks in this graph at 1.2 and $2.4 \mathrm{~Hz}$ are typical harmonics of the rotor speed ( 1.2 revolutions per second) and are probably deterministic rotor responses such as those induced by tower passage and gravity loading along the blade. The first and second flapwise natural frequency modes are indicated by broad peaks around $3.25 \mathrm{~Hz}$ and $9.0 \mathrm{~Hz}$, respectively.

For a more detailed discussion of the analysis of the Northern Power 100-kW wind turbine, see References 3 and 4. Data analysis methods and specific techniques used in WINDATS were taken from References 5-9.

\section{CONCLUSIONS}

This analysis package has proven to be a useful tool to efficiently process large amounts of test data in a timely manner. Although researchers have started using this analysis package in the office and in the field, it is still in the development stage. We plan to add a few more operations, such as the choice of a least squares data fit in place of azimuth averaging, using a Fourier series with several low-frequency terms to produce a model of the deterministic part of the data. Subtracting the curve fit from the original time-series data leaves residuals as before (the stochastic part), representing the contributions from wind turbulence. Several operations related to spectral analysis will also be added, such as computing the integrated area under the power spectral density curve to yield the variance of the signal over the bandwidth of the frequency range of interest. Frequency weighting, normalization, and transfer and correlation functions would also be useful for complete frequency domain analysis capabilities.

\section{ACRHOWLEDGMENTS}

This work was sponsored by the U.S. Department of Energy under contract number DE-AC02-83CH10093. Bob Thresher, Neil Kelley, and George Scott provided algorithmic assistance; Marshall Buhl provided computational assistance; and Richard Osgood, Alan Wright, and Sandy Butterfield provided users' feedback.

\section{REFERENCES}

[1] R. Sharif-Razi, R. Thresher, and W.E.Holley, Digital Stimulation of Turbulence Excitation for Dynamic Analysis of Wind Turbines, Mechanical Engineering Department, Oregon State University, Corvallis, NASA Grant NAG-3-87, March, 1985.

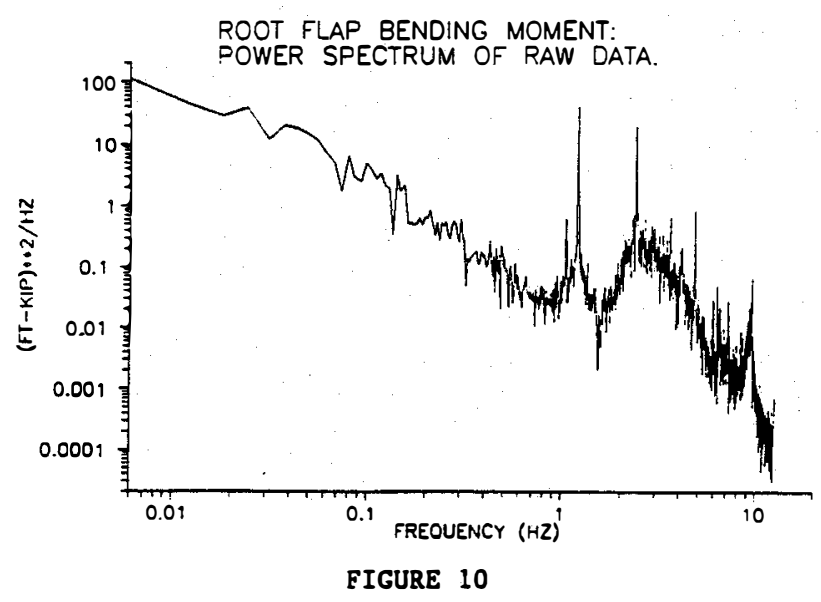

[2] D.E. Newland, Random Vibrations and Spectral Analysis, Longman Inc., New York, 1975.

[3] R.M. Osgood and S.M. Hock, Preliminary Results from the Dynamic Response Testing of the Northern Power Systems 100-kW Wind Turbine, SERI/ TP-217-3342, Solar Energy Research Institute, Golden, Colorado, June 1988.

[4] S.M. Hock, R.W. Thresher, and A.D. Wright, $A$ Comparison of Results from Dynamic Response Field Tests, SERI/TP-217-3423, Solar Energy Research Institute, Golden, Colorado, November 1988.

[5] A.C. Hansen, Wind Turbine Dynamic Data Analysis Solftware User's Guide (and software), Mechanical Engineering. Department, University of Utah, Salt Lake City, SERI Subcontract No. XL-605078-2, October 1987.

[6] P.H. Madsen, DAP-1, A Data Analysis Package for Spectral Analysis, Version 1.2, Wind Energy Research Center, Solar Energy Research Institute, Golden, Colorado, No. 217/Draft/ WRB1075/11-6-87.

[7] R.K. Otnes and L. Enochson, Applied Time Series Analysis, Vol. 1, J. Wiley and Sons, New York, 1978.

[8] S.D. Stearns and R.A. David, Signal Processing Algorithms, Prentice-Hall, Inc., Englewood Cliffs, New Jersey, 1988.

[9] W.H. Press, B.P. Flannery, S.A. Teukolsky, and W.T. Vetterling, Numerical Recipes, The Art of Scientific Computing, Cambridge University Press, New York, 1986. 